\title{
BMJ Open Laparoscopic excision of deep rectovaginal endometriosis in BSGE endometriosis centres: a multicentre prospective cohort study
}

\author{
Dominic Byrne, ${ }^{1}$ Tamara Curnow, ${ }^{2}$ Paul Smith, ${ }^{3}$ Alfred Cutner, ${ }^{4}$ Ertan Saridogan, ${ }^{4}$ \\ T Justin Clark, ${ }^{5}$ on behalf of BSGE Endometriosis Centres
}

To cite: Byrne D, Curnow T, Smith P, et al. Laparoscopic excision of deep rectovaginal endometriosis in BSGE endometriosis centres: a multicentre prospective cohort study. BMJ Open 2018;8:e018924. doi:10.1136/ bmjopen-2017-018924

- Prepublication history and additional material for this paper are available online. To view these files, please visit the journal online (http://dx.doi. org/10.1136/bmjopen-2017018924).

Received 1 September 2017 Revised 10 November 2017 Accepted 27 November 2017

Check for updates

${ }^{1}$ Royal Cornwall Hospitals Trust, Truro, UK

${ }^{2}$ Bodriggy Health Centre, Hayle, UK

${ }^{3}$ Birmingham Women's NHS Foundation Trust, School of Clinical and Experimental Medicine, Birmingham, UK ${ }^{4}$ University College London Hospitals, London, UK

${ }^{5}$ Birmingham Women's NHS Foundation Trust, University of Birmingham, Birmingham, UK

Correspondence to Professor T Justin Clark; t.j.clark@doctors.org.uk

\section{ABSTRACT}

Objective To estimate the effectiveness and safety of laparoscopic surgical excision of rectovaginal endometriosis.

Design A multicentre, prospective cohort study. Setting 51 hospitals accredited as specialist endometriosis centres.

Participants 5162 women of reproductive age with rectovaginal endometriosis of which 4721 women had planned laparoscopic excision.

Interventions Laparoscopic surgical excision of rectovaginal endometriosis requiring dissection of the pararectal space.

Main outcome measures Standardised symptom questionnaires enquiring about chronic pelvic pain, bladder and bowel symptoms, analgesia use and quality of life (EuroQol) completed prior to surgery and at 6,12 and 24 months postoperatively. Serious perioperative and postoperative complications including major haemorrhage, infection and visceral injury were recorded.

Results At 6 months postsurgery, there were significant reductions in premenstrual, menstrual and non-cyclical pelvic pain, deep dyspareunia, dyschezia, low back pain and bladder pain. In addition, there were significant reductions in voiding difficulty, bowel frequency, urgency, incomplete emptying, constipation and passing blood. These reductions were maintained at 2 years, with the exception of voiding difficulty. Global quality of life significantly improved from a median pretreatment score of 55/100 to 80/100 at 6 months. There was a significant improvement in quality of life in all measured domains and in quality-adjusted life years. These improvements were sustained at 2 years. All analgesia use was reduced and, in particular, opiate use fell from $28.1 \%$ prior to surgery to $16.1 \%$ at 6 months. The overall incidence of complications was $6.8 \%(321 / 4721)$. Gastrointestinal complications (enterotomy, anastomotic leak or fistula) occurred in $52(1.1 \%)$ operations and of the urinary tract (ureteric/ bladder injury or leak) in $49(1.0 \%)$ procedures.

Conclusion Laparoscopic surgical excision of rectovaginal endometriosis appears to be effective in treating pelvic pain and bowel symptoms and improving health-related quality of life and has a low rate of major complications when performed in specialist centres.
Strengths and limitations of this study

- Our study is by far the largest, multicentre observational cohort published for the laparoscopic surgical treatment of rectovaginal endometriosis with a sample of nearly 5000 cases.

- Data were prospectively collected, minimising missing data and recall bias, and were obtained from multiple centres enhancing transferability, and outcomes measurements were patient reported reducing interpreter bias.

- Efficacy outcomes were assessed in both the short term (at 6 months) and longer term (at 2 years) following surgery. In addition, the scale of these data and the method of collection have enabled a robust assessment of the risk of complications from this type of surgery. The reported incidence of complications cannot, however, be used as indicative risk for patients who have care given in non-specialist endometriosis centres.

- The main limitation of our study relates to missing data from incomplete data entry, incomplete follow-up or uncompleted follow-up at closure of the study. We performed sensitivity analyses to explore the robustness of our results to incompleteness. The results were stable, remaining significant in some cases even when symptomatic outcomes for those women with missing data were assumed to be the worst possible outcome.

- Historical control data were used from the same patients prior to surgical intervention, although the study would have benefited from a non-surgically treated control group. However, persuading a group of women with severe, refractory symptoms to continue with non-surgical treatment would be challenging.

\section{INTRODUCTION}

Endometriosis is a common and serious problem for women in their reproductive years and can cause chronic pelvic pain, subfertility and bowel and urinary dysfunction. ${ }^{1}$ The associated morbidity places a substantial economic burden on society as a 
result of direct healthcare costs and indirect productivity losses. In the USA, direct healthcare costs have been estimated to be US $\$ 2801$ annually per patient with an additional cost of US $\$ 1023$ annually per patient due to loss of productivity. Overall, the costs associated with endometriosis in the USA are estimated at US $\$ 22$ billion per annum. $^{2}$

Deep endometriosis in the posterior pelvis frequently affects the space between the anterior wall of the rectosigmoid and the posterior vaginal wall and is usually referred to as rectovaginal endometriosis. There is limited evidence supporting the sustained effectiveness and acceptability of medical therapies in improving the symptoms of rectovaginal endometriosis. ${ }^{3-5}$ Consequently, surgical treatment has been proposed to completely excise the deep rectovaginal disease. ${ }^{6-8}$

Advances in instrumentation and surgical experience have led to laparoscopic treatment superseding alternative surgical routes such as laparotomy and transvaginal excision. Previous studies have reported improvements in generic quality of life (QoL) data following surgical excision of endometriosis involving the bowel, but these evaluations have been undertaken using small cohorts of women usually from single centres, affecting the precision and generalisability of the derived results. ${ }^{9-11}$ While these findings of improved symptomatic outcomes are promising, it is well recognised that surgery for deep endometriosis with bowel involvement is complex and can be associated with serious and potentially life threatening complications. ${ }^{10}$ For these reasons, the European Society of Human Reproduction and Embryology (ESHRE) Guidelines on the 'Management of women with endometriosis' recommend that clinicians refer women with suspected or diagnosed deep endometriosis to a centre of expertise that offers all available treatments in a multidisciplinary context. ${ }^{1}$

In 2006, the British Society for Gynaecological Endoscopy (BSGE) developed specialist endometriosis centres (Endocentres), where patients would be treated by surgeons who work in multidisciplinary teams, audit their outcomes and perform sufficient workload to maintain their surgical skills. ${ }^{12}$

In view of the paucity of world literature data pertaining to the effectiveness and safety of this highly complex surgery for a common gynaecological condition, we undertook a prospective, multicentre cohort study to estimate (1) effectiveness of surgery on patient reported symptoms associated with endometriosis as well as its impact on women's health-related QoL and (2) safety by examining rates of surgical complications using data collected from the BSGE Endocentres dataset.

\section{METHODS}

\section{Study design}

A multicentre prospective cohort study of premenopausal women undergoing surgery for pelvic pain associated with rectovaginal disease resistant to medical treatment or conservative surgical therapy was performed. Standardised diagnostic, operative, histological and patient outcome data were prospectively collected from 51 BSGE Endocentres between 1 January 2009 and 30 June 2016.

\section{Study population}

Women treated in a BSGE Endocentre who underwent laparoscopic excision of deep rectovaginal endometriosis, which required dissection of the pararectal space and gave written consent for data collection, were included in the study. Dissection of the pararectal space was chosen as the inclusion criterion for cases to be studied because access to this anatomical space is necessary to free adherent bowel prior to excision of deep rectovaginal disease. Furthermore, this operative step is necessary irrespective of the type of surgery performed on the bowel. So by choosing this step of the surgical procedure, the BSGE could be assured that all cases of deep rectovaginal endometriosis would be included reducing the risk of selection bias. Clear explanation of dissection of the deep pararectal space is provided on the BSGE endometriosis database for surgeons. While there have been a number of historical scoring systems for endometriosis, some were not developed prior to the inception of the BSGE project and none have been universally accepted. ${ }^{13-16}$ In order to retain accreditation as a BSGE Endocentre, all consenting patients who undergo surgery for deep rectovaginal endometriosis that includes dissection of the pararectal space in an Endocentre must have their data entered on to the BSGE national endometriosis database.

\section{Clinical data}

Standardised patient symptom data and QoL assessments were collected prior to surgery as a baseline control. The assessments were repeated at 6 months, 1 year and 2 years after surgery. To ensure consistent timing of follow-up, the database only accepts postoperative data entry within an interval from 4 weeks before the exact date required and up to 8 weeks after the exact date.

All patients recorded their clinical symptoms on a BSGE standard questionnaire using a $0-10$ point Likert scale for premenstrual pain, menstrual pain, non-cyclical pelvic pain, deep dyspareunia, cyclical dyschezia, non-cyclical dyschezia, lower back pain, bladder pain and voiding difficulty. In addition, patients recorded details of bowel function with graded answers for: frequency of bowel movement, urgency of bowel movement, incomplete empyting sensation, constipation and blood in the stool. Patient-reported QoL data were collected using EuroQol 5D-3L (EQ-5D-3L) questionnaire and EuroQol Visual Analogue Score. ${ }^{17} 18$ The five domains of the EQ-5D-3L questionnaire were combined to compute a single weighted index score (a more sensitive measure of QoL) using the standard UK time trade-off health-state valuation ${ }^{19}$ (as used by National Institute for Health and Care Excellence for assessing quality-adjusted life years (QALYs)). In this EQ-5D index score, 1 represents full health and 0 represents death. There 
are some health states that are assigned negative values (ie, worse than death).

Dichotomous data ('yes or 'no') were collected for use of analgesia (paracetamol, non-steroidal anti-inflammatory drug (NSAID) or opiates) and medical therapy (contraceptive pill/oral contraceptives, Mirena Intrauterine system (Bayer, Germany), gonadotrophin releasing hormone (GNRH) analogues alone, GNRH analogues plus add-back hormone replacement, systemic progestogens or aromatase inhibitors).

\section{Surgical data}

Details of previous endometriosis surgery were recorded including adnexal surgery and hysterectomy. Surgical details were collected using a standard dataset describing the name and level of the surgeon, whether a colorectal and/or a urological surgeon also undertook the surgery and whether the surgery was laparoscopic or laparotomic. The distribution of any endometriosis deposits was described by 'yes' or 'no', for right and left pelvic side wall, right and left endometrioma, right and left uterosacral ligament and obliteration of the pouch of Douglas. Bowel involvement of endometriosis was also recorded dichotomously for 'rectal involvement', 'rectovaginal nodule' or involvement of 'appendix', 'small bowel' and 'rectosigmoid'. Coexistent bladder endometriosis was recorded by yes or no for superficial bladder, deep bladder and deep uterovesical endometriosis.

The surgeon recorded the surgical procedure for each of the above areas of distribution of endometriosis from a list of 'ablated', 'excised', 'ablated and excised', 'not treated' or 'not present'. Surgery on any endometrioma present was recorded by selecting from a list of 'ablated', 'excised', 'oophorectomy', 'drained only', 'not treated' or 'not applicable'. Pararectal space dissected was recorded as yes or no. Surgery on a rectovaginal nodule was recorded as 'ablated', 'excised', 'not treated' or 'not applicable'. Opening of the vagina as part of the surgery was recorded as 'yes', 'no' or 'not applicable'. The type of bowel surgery was recorded by selecting from a list of 'not applicable', 'not treated', 'shaved', 'disc resection' or 'segmental resection', along with whether a stoma was formed or not. Surgery on any bladder endometriosis was recorded as 'ablated', 'excised without bladder opening', 'excised with bladder opening', 'not present' or 'not treated'. Ureteric endometriosis surgery was described by ureteric nodule excised (yes, no or not present), right ureterolysis (yes, no or not applicable), left ureterolysis (yes, no or not applicable), JJ stent (yes or no). Finally, data were collected regarding performance of a concomitant hysterectomy (yes, no or not applicable) with space for free text.

Recording of complications was divided into two sections with 'yes' or 'no' answers for a standardised list of perioperative and postoperative complications. Complication data included surgical injury to urological, gastrointestinal or vascular structures, unplanned procedures including conversion to laparotomy, infective morbidity, pulmonary embolism and death. Histology of removed specimens was examined for malignant transformation.

\section{Statistical methods}

Data were analysed according to the study eligibility criteria, namely: a valid operation date was entered, the intended operation was via a laparoscopy, the pararectal space was dissected and there was excision of endometriosis. If duplicate data were present, the most complete dataset was used. Centres that entered fewer than 20 cases in the total study period were excluded.

Non-numerical scores (eg, bowel symptoms) and the EQ-5D-3L were coded numerically with an assumed underlying interval scale. Median scores were computed along with the $95 \%$ CI about the median.

For the patient-reported data, pair-wise comparisons were made using the baseline preoperative data as a control. Data were thus only included if both the preoperative and the relevant postoperative data were entered. We also compared the symptom scores at 6 months with those at 2 years to assess the postoperative trend. The Mann-Whitney U-test (Wilcoxon rank sum) was used for comparison for data with more than two outcomes and the sign test for dichotomous data. A statistic was considered significant if the probability of it occurring by chance was $<0.05$ on a two-tailed test.

As postoperative follow-up was incomplete, the impact of this was assessed in two ways. The first method was by restricting the analysis to centres with more complete follow-up, defined as centres performing at least 50 operations in the study period, with more than $90 \%$ of preoperative questionnaires and more than $70 \%$ of postoperative questionnaires entered onto the BSGE database for at least one postoperative clinical follow-up period. The second method was to include all women for whom a preoperative, baseline score was present. If the postoperative results were reported, then these were used. If the postoperative results were not present, then an assigned score was used. The assigned score was the same for all patients with missing postoperative data. The assigned score was initially the best outcome possible and was then changed stepwise through less desirable outcomes. With each assigned value, the significance calculation was repeated. The last value of the assigned score at which the outcome is still significant gives a measure of the sensitivity of the result to the missing data. If, for example, the statistic is still significant with the worst possible outcome assigned to the missing postoperative dataset, then the outcome is effectively independent of the missing responses.

The data analysis was performed using Matlab (MathWorks) V.R2011b.

\section{RESULTS}

In BSGE Endocentres between 1 January 2009 and 30 June 2016, 5162 women underwent surgery for deep rectovaginal endometriosis, which included dissection 
of the pararectal space. Women who underwent planned laparotomy $(160 ; 3.1 \%)$, only had ablative treatment of their endometriosis $(100 ; 1.9 \%)$ or had no treatment of their endometriosis $(181 ; 3.5 \%)$ were excluded from further analysis. Thus, a total of 4721 women had planned laparoscopic excision of deep rectovaginal endometriosis in a total of 51 Endocentres. Previous surgery for endometriosis had been performed in $55.1 \%$ (2602) of these women, with $7.0 \%$ (333) having had one ovary removed, $3.0 \%$ (141) both ovaries removed and $5.0 \%$ (234) having had a hysterectomy. The median age of women having surgery was 35.1 years (90th centile range 25.9-44.8 years).

\section{Surgical findings and procedures}

At surgery, endometriosis was identified on the left pelvic side wall in $69.0 \%$ (3259) of patients and on the right side wall in $57.7 \%$ (2726). It was on the left uterosacral ligament in $78.4 \%$ (3702) patients and the right uterosacral ligament in $70.8 \%$ (3341). The pouch of Douglas was obliterated in $67.1 \%$ (3167) women, and a rectovaginal nodule was present in $68.6 \%$ (3238) women. There was endometriosis present on the rectum in $54.7 \%$ (2582) women, the caecum in $1.3 \%$ (60) women, the appendix in $2.3 \%$ (110) women, small bowel in $1.6 \%$ (75) women and rectosigmoid in $18.1 \%$ (856) women. Deep uterovesical disease was present in 422 women $(8.9 \%)$.

Gonadatrophin-releasing hormone agonists were given to $23.0 \%$ (1087) women prior to surgery. The operation was undertaken by a senior gynaecologist in $96.4 \%$ (4549) cases, and a colorectal surgeon was present in $27.6 \%$ (1304) cases and a urologist in 320 (6.8\%) cases. Pararectal dissection was performed in all cases and ureterolysis on the left in $65.5 \%$ (3092) and on the right in $57.1 \%$ (2695) cases. A ureteric nodule of endometriosis was excised in $9.0 \%$ (424) and JJ ureteric stents used in $9.2 \%$ (434) cases. Bowel surgery was performed in $63.1 \%$ (2981) cases, and $1.3 \%$ (62) women had a stoma. A hysterectomy was performed in $723(15.3 \%)$ women. Conversion to laparotomy occurred in $41(0.9 \%)$ cases. There were no cases of malignancy in any of the endometriosis specimens.

\section{Follow-up performance}

While preoperative data were expected in 4721 women, symptom data were available for $4210(89 \%)$ and QoL data for 4041 (86\%) women. At 6-month follow-up symptom, scores were available in $2350(50 \%)$ and QoL scores in 2241 (47\%) of women. At 1 year postoperation, data were expected for 3977 women, of whom symptom data were present in 1499 (38\%) and QoL in 1380 (35\%). At 2 years, symptom data were present in $729(27 \%)$ and QoL data in 644 (24\%) out of a possible 2704 women.

In the seven Endocentres with most complete follow-up, preoperative symptom data were available for $684(97 \%)$ of the 707 women and QoL data for $668(94 \%)$ women. At 6 months, symptom data were present for $537(76 \%)$ and QoL data for $530(75 \%)$ women. At 1 year postsurgery, data were expected from 553 women, and symptom data were present in $356(64 \%)$ and QoL in 347 (63\%) women. At 2 years postoperation, data were expected in 319 women, and symptom data were present for 160 $(50 \%)$ and QoL data in $145(45 \%)$ women.

\section{Symptom outcomes}

At 6 months after laparoscopic excision of endometriosis, there was a significant reduction in pain scores for premenstrual pain (from a median of $7 / 10$ to $3 / 10$ ), menstrual pain (from $9 / 10$ to $5 / 10$ ) and non-cyclical pelvic pain (from $6 / 10$ to $2 / 10$ ) when compared with preoperative scores. A significant reduction in pain scores also occurred for deep dyspareunia (from 6/10 to $1 / 10)$, cyclical $(6 / 10$ to $1 / 10)$ and non-cyclical dyschezia $(3 / 10$ to $0 / 10)$, low back pain $(6 / 10$ to $3 / 10)$ and a statistically significant drop in bladder pain although no change in the median score. In addition, there was a statistically significant (although not clinically significant) reduction in voiding difficulty, bladder pain, bowel frequency, bowel urgency, incomplete bowel emptying, constipation and passing blood in the stool (see table 1).

The same statistically significant reduction in symptoms remained present at 2 years postsurgery for all symptoms except voiding difficulty. A comparison of the symptoms at 6 months was made with the symptoms at 2 years in order to assess the postoperative trend in symptom scores. This showed that there was a statistically significant but clinically small increase in all symptoms except voiding difficulty over the 18 months (see table 1 ).

The median global QoL on a 100-point visual analogue scale improved from 55 preoperatively to 80 postoperatively. There was no statistically significant degradation in effect between 6 and 24 months in all measured QoL domains with the exception of the visual analogue score, which showed a statistically significant (although clinically negligible) lessening over this timeframe (see table 2, median score dropped from 80/100 to 76/100). Patient-reported QoL for all five domains of the EuroQol questionnaire showed a statistically significant improvement in QoL at 6 months postsurgery that was sustained at 2 years postsurgery. These five domains are combined to give a single weighted index score (EQ5D index) that can be used as a measure of QALYs. This more sensitive measure showed a clinically significant improvement (from a mean of 0.525 to 0.756 ) at 6 months postsurgery that was sustained at 2 years $(0.751)$.

Analgesia use was significantly reduced at 6 and 24 months postsurgery compared with preoperative levels for all three analgesic types. Paracetamol use dropped from $76.0 \%$ patients to $59.8 \%$ at 6 months, NSAID use dropped from $69.8 \%$ patients to $48.9 \%$ at 6 months and opiate use dropped from $28.1 \%$ patients to $16.1 \%$ at 6 months. There was however a statistically significant (although clinically small) increase in paracetamol, NSAID and opiate use between 6 and 24 months postoperatively (see table 3 ). 
Table 1 Patient-reported symptoms prior to laparoscopic surgical excision of rectovaginal endometriosis and at 6 months, 1 year and 2 years post-treatment (median scores are presented (0-10 for the first nine symptoms and 0-4 for the remaining five symptoms) with total number of responses in round brackets and $95 \% \mathrm{Cl}$ of the median in square brackets)

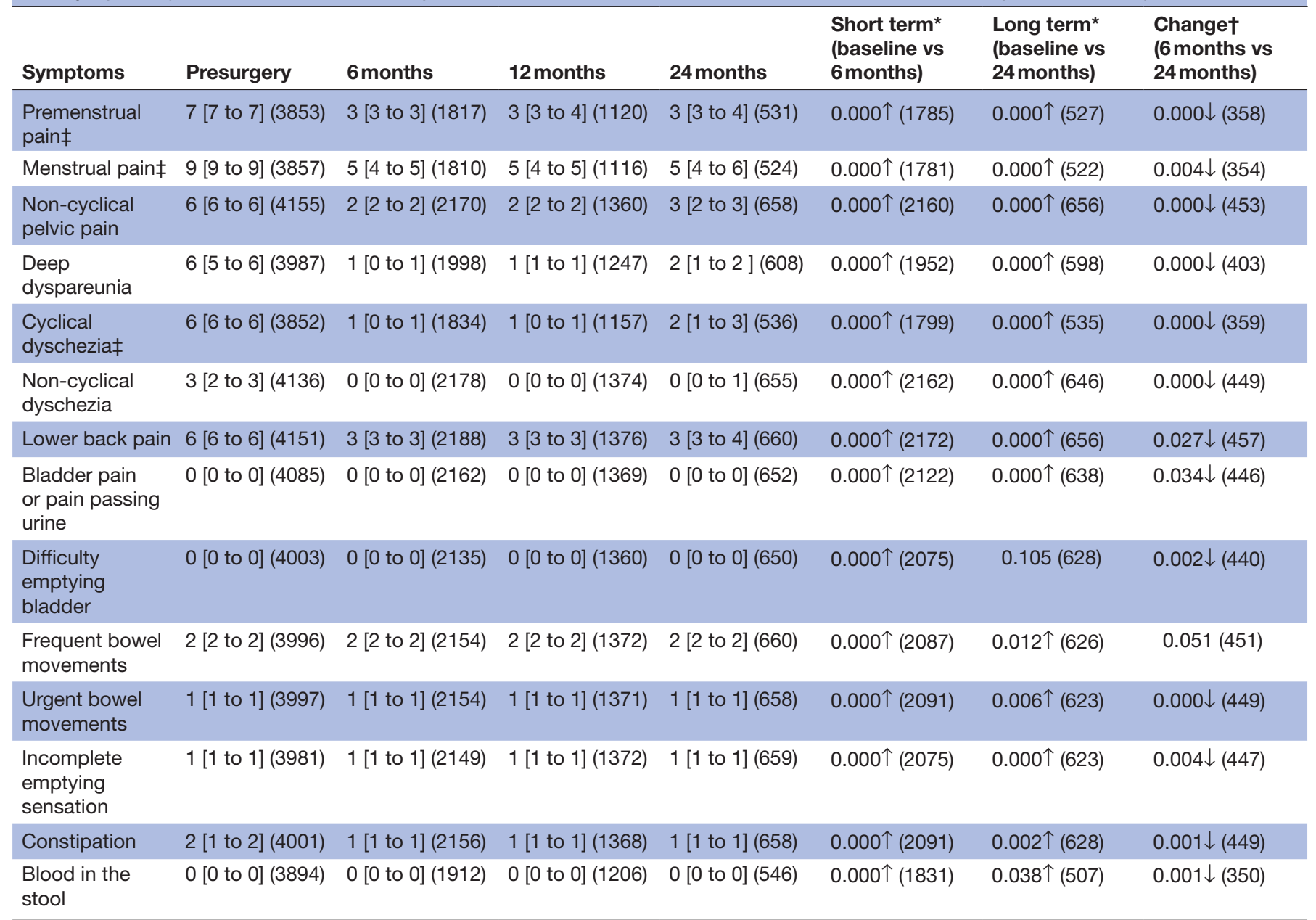

A lower score is associated with less severe symptoms.

Note that there is some variation in total number of responses depending on whether patients chose not to answer some questions.

*Statistical comparison (Mann-Whitney U test) of short-term symptom scores (6 months postsurgery) and long-term symptom scores (2 years postsurgery) with baseline scores (presurgery).

†Statistical comparison (Mann-Whitney U test) of change in symptom scores over time post-treatment (between 6 months and 2 years).

‡Excludes patients who had a hysterectomy prior to this surgery.

$\uparrow$, statistically significant increase; $\downarrow$, statistically significant decrease.

\section{Sensitivity analyses}

Sensitivity analysis on the reduction of patient reported symptoms and the improvement in QoL postsurgery was used to estimate the effect of incomplete data on the significance of the results and is shown in table 4. For all symptoms except voiding difficulty, constipation and blood in the stool, the median scores for missing postoperative data at 6 months would have to be higher (worse) than at baseline for change in scores to become statistically non-significant. Indeed, menstrual pain scores at 6 months remained significantly improved on the preoperative scores even if all the missing 6-month scores are assumed to be 10 (maximum pain). To further test the robustness of the data to missing values, data from the seven centres with the best follow-up were analysed separately (see table 5, supplementary tables 1-3). Improvement in premenstrual pain, menstrual pain, non-cyclical pelvic pain, deep dyspareunia, cyclical dyschezia, lower back pain, EuroQol Visual Analogue Scale (EQVAS) and EQ5D index were all statistically significant even if the missing data scores were of the worst possible outcome.

\section{Surgical complications}

The overall incidence of complications was $6.8 \%$ (321), with perioperative complications in $4.7 \%$ (220) operations and late operative complications in $2.5 \%$ (120) women, including 19 women suffering both perioperative and postoperative complications (see table 6). Bowel complications occurred in $1.1 \%$ (52 operations) and the incidence varied according to whether bowel surgery was undertaken and, if so, what type of procedure; $0.6 \%$ (11) where no coexistent bowel surgery was undertaken, $1.1 \%$ (29) with bowel shaving, $9.3 \%$ (5) with disc resection and $3.9 \%$ (7) with segmental bowel resection (see table 7). 
Table 2 Median scores for patient-reported quality of life prior to treatment and at 6 months, 1 year and 2 years post-treatment with total number of patients in round brackets and $95 \%$ Cls in square brackets

\begin{tabular}{|c|c|c|c|c|c|c|c|}
\hline Quality of life & Presurgery & 6 months & 12 months & 24 months & $\begin{array}{l}\text { Short term* } \\
\text { (baseline vs } \\
6 \text { months) }\end{array}$ & $\begin{array}{l}\text { Long term* } \\
\text { (baseline vs } \\
24 \text { months) }\end{array}$ & $\begin{array}{l}\text { Change } \\
\text { (6 months vs } \\
24 \text { months) }\end{array}$ \\
\hline EQVAS numeric $\neq$ & $\begin{array}{l}55[55 \text { to } 57] \\
(4015)\end{array}$ & $\begin{array}{l}80[75 \text { to } 80] \\
(2050)\end{array}$ & $\begin{array}{l}80 \text { [75 to } 80] \\
(1247)\end{array}$ & $\begin{array}{l}76[75 \text { to } \\
80](575)\end{array}$ & $0.000 \downarrow(2045)$ & $0.000 \downarrow(573)$ & $0.024 \uparrow(396)$ \\
\hline $\begin{array}{l}\text { EQ5D usual } \\
\text { Activities }\end{array}$ & 1 [1 to 1$]$ (4005) & 0 [0 to 0$]$ (2051) & 0 [0 to 0$]$ (1250) & 0 [0 to 0$](574)$ & $0.000 \uparrow(2042)$ & $0.000 \uparrow(570)$ & 0.477 (395) \\
\hline $\begin{array}{l}\text { EQ5D anxiety } \\
\text { depression }\end{array}$ & 1 [1 to 1] (3991) & 0 [0 to 0$]$ (2049) & 0 [0 to 0$]$ (1252) & 0 [0 to 0$](573)$ & $0.000 \uparrow(2033)$ & $0.000 \uparrow(569)$ & $0.331(395)$ \\
\hline EQ5D mobility & 0 [0 to 0$]$ (3999) & 0 [0 to 0$]$ (2046) & 0 [0 to 0$]$ (1250) & 0 [0 to 0$]$ (569) & $0.000 \uparrow(2035)$ & $0.000 \uparrow(563)$ & 0.339 (392) \\
\hline EQ5D self-care & 0 [0] (3994) & 0 [0 to 0$]$ (2033) & 0 [0 to 0$]$ (1248) & 0 [0 to 0$](558)$ & $0.000 \uparrow(2020)$ & $0.001 \uparrow(548)$ & $0.364(384)$ \\
\hline EQ5D index $¥ \S$ & $\begin{array}{l}0.689[0.689 \text { to } \\
0.689](3966)\end{array}$ & $\begin{array}{l}0.796[0.796 \text { to } \\
0.796](2032)\end{array}$ & $\begin{array}{l}0.796[0.796 \text { to } \\
0.796](1245)\end{array}$ & $\begin{array}{l}0.796[0.796 \text { to } \\
0.796](556)\end{array}$ & $0.000 \downarrow$ (2010) & $0.000 \downarrow(546)$ & $0.043 \uparrow(383)$ \\
\hline
\end{tabular}

Median EQVAS lies in the range 0-100 with a higher score associated with a better quality of life. The other median scores (EQ5D) lie in the range $0-2$, where a lower score is associated with a better quality of life.

Note that there is some variation in total number of responses depending on whether patients chose not to answer some questions.

*Statistical comparison (Mann-Whitney U test) of short-term symptom scores (6 months postsurgery) and long-term symptom scores (2 years postsurgery) with baseline scores (presurgery).

†Statistical comparison (Mann-Whitney $U$ test) of change in symptom scores over time post-treatment (between 6 months and 2 years). $\ddagger A$ high EQVAS score or a high EQ5D index score is associated with an improvement in QoL, whereas with all other QoL and symptom scores, a low value is associated with improvement.

$\S E Q 5 D$ index of 1 is equivalent to perfect health and 0 equivalent to death. Means are reported rather than medians.

$\uparrow$, statistically significant increase; $\downarrow$, statistically significant decrease; EQVAS, EuroQol Visual Analogue Scale; EQ5D, EuroQol 5D; QoL, quality of life.

\section{DISCUSSION}

\section{Statement of principal findings}

Laparoscopic excision of severe rectovaginal endometriosis, performed in specialist centres in women with chronic pelvic pain, was associated with significant reduction in pain symptoms and improved health-related QoL. Moreover, the reduction in pain and increased QoL observed 6 months following surgery was maintained at 2 years. All types of pain symptoms improved: premenstrual pain, menstrual pain, non-cyclical pain, back pain, pain with sexual intercourse, pain on voiding and on opening the bowels. A significant reduction in the need for analgesia supported the findings of an overall reduction in pain symptoms.
Bowel symptoms including frequency, urgency, incomplete emptying and constipation also improved. This type of surgery requires enhanced laparoscopic skills, primarily because of the need to overcome distorted anatomy and operate in proximity to delicate gastrointestinal, genitourinary and vascular structures. However, clinical outcomes were good, and the rates of serious perioperative and late complications were low, when laparoscopic excision of rectovaginal endometriosis was conducted in recognised, specialist centres.

\section{Strengths and weaknesses of the study}

Our study has many strengths that include the size of the sample with nearly 5000 cases, the largest dataset by

Table 3 Analgesia use prior to surgery and at 6 months, 1 year and 2 years postsurgery

\begin{tabular}{|c|c|c|c|c|c|c|c|}
\hline Analgesia & Presurgery & 6 months & 12 months & 24 months & $\begin{array}{l}\text { Short term* } \\
\text { (baseline vs } \\
6 \text { months) }\end{array}$ & $\begin{array}{l}\text { Long term* } \\
\text { (baseline vs } \\
24 \text { months) }\end{array}$ & $\begin{array}{l}\text { Change } \dagger \\
\text { (6 months vs } \\
24 \text { months) }\end{array}$ \\
\hline Paracetamol & $\begin{array}{l}76.0 \% \\
(4118)\end{array}$ & $\begin{array}{l}59.8 \% \\
(1934)\end{array}$ & $\begin{array}{l}60.6 \% \\
(1235)\end{array}$ & $61.4 \%(610)$ & $0.000 \uparrow(1915)$ & $0.000 \uparrow(604)$ & $0.001 \downarrow$ (388) \\
\hline NSAID & $\begin{array}{l}69.8 \% \\
(4099)\end{array}$ & $\begin{array}{l}48.9 \% \\
(1924)\end{array}$ & $\begin{array}{l}48.3 \% \\
(1229)\end{array}$ & $52.0 \%(603)$ & $0.000 \uparrow(1903)$ & $0.000 \uparrow(593)$ & $0.013 \downarrow$ (385) \\
\hline Opiates & $\begin{array}{l}28.1 \% \\
(3953)\end{array}$ & $\begin{array}{l}16.1 \% \\
(1895)\end{array}$ & $\begin{array}{l}16.8 \% \\
(1214)\end{array}$ & $16.6 \%(592)$ & $0.000 \uparrow(1859)$ & $0.000 \uparrow(575)$ & $0.006 \downarrow$ (376) \\
\hline
\end{tabular}

Note that there is some variation in total number of responses depending on whether patients chose not to answer some questions. *Statistical comparisons of short-term change in analgesia use (6 months postsurgery) and long-term change in analgesia use (2 years postsurgery) with baseline analgesia use (presurgery) assessed using a two-tailed sign test.

†Statistical comparison of change in analgesia use over time post-treatment (between 6 months and 2 years) using a two-tailed sign-test. $\uparrow$, statistically significant increase; $\downarrow$, statistically significant decrease; NSAID, non-steroidal anti-inflammatory drug. 
Table 4 Sensitivity analysis for missing postoperative data

Short term Long term

(6 months)* (2years)* Range

\begin{tabular}{|c|c|c|c|}
\hline \multicolumn{4}{|l|}{ Symptoms } \\
\hline Premenstrual pain & 9 & 8 & $(0-10)$ \\
\hline Menstrual pain & 10 & 10 & $(0-10)$ \\
\hline $\begin{array}{l}\text { Non-cyclical pelvic } \\
\text { pain }\end{array}$ & 7 & 6 & $(0-10)$ \\
\hline Deep dyspareunia & 8 & 6 & $(0-10)$ \\
\hline Cyclical dyschezia & 7 & 7 & $(0-10)$ \\
\hline $\begin{array}{l}\text { Non-cyclical } \\
\text { dyschezia }\end{array}$ & 5 & 3 & $(0-10)$ \\
\hline Low back pain & 7 & 7 & $(0-10)$ \\
\hline Bladder pain & 2 & 1 & $(0-10)$ \\
\hline Voiding difficulty & 0 & NS & $(0-10)$ \\
\hline $\begin{array}{l}\text { Frequent bowel } \\
\text { movements } †\end{array}$ & 3 & 3 & $(0-4)$ \\
\hline $\begin{array}{l}\text { Urgent bowel } \\
\text { movements } †\end{array}$ & 2 & 2 & $(0-4)$ \\
\hline $\begin{array}{l}\text { Incomplete bowel } \\
\text { movements } \dagger\end{array}$ & 2 & 2 & $(0-4)$ \\
\hline Constipation† & 2 & 2 & $(0-4)$ \\
\hline Blood in the stoolt & 0 & 1 & $(0-4)$ \\
\hline \multicolumn{4}{|l|}{ Quality of life $†$} \\
\hline $\begin{array}{l}\mathrm{EQ} \text { visual } \\
\text { analogue score }\end{array}$ & 40 & 55 & $(0-100)$ \\
\hline $\begin{array}{l}\text { EQ5D usual } \\
\text { activities }\end{array}$ & 2 & 1 & $(0-2)$ \\
\hline $\begin{array}{l}\text { EQ5D pain and } \\
\text { discomfort }\end{array}$ & 2 & 2 & $(0-2)$ \\
\hline $\begin{array}{l}\text { EQ5D anxiety and } \\
\text { depression }\end{array}$ & 1 & 1 & $(0-2)$ \\
\hline EQ5D mobility & 1 & 1 & $(0-2)$ \\
\hline EQ5D self-care & 1 & 1 & $(0-2)$ \\
\hline EQ5D index & 0.45 & 0.65 & $(-0.594$ to 1$)$ \\
\hline
\end{tabular}

*The worst possible score that could be reported for all the missing postoperative data in order for the short-term (6 months) or longterm statistics in tables 3 and 4 to still be significant. (The test statistics that were not significant (NS) to start with have not been included.)

†A high EQVAS or EQ5D index score is associated with an improvement in QoL, whereas with all other QoL and symptom scores, a low value is associated with improvement.

EQ5D, EuroQol 5D; EQVAS, EuroQol Visual Analogue Scale; QoL, quality of life.

far reported to date in the world literature. These data were collected prospectively, minimising missing data and recall bias, and were obtained from multiple centres enhancing transferability, and outcome measurements were patient-reported reducing interpreter bias (online supplementary file). Efficacy outcomes were assessed in both the short term (at 6 months) and longer term (at 2 years) following surgery. In addition, the scale of these
Table 5 Sensitivity analysis for missing postoperative data restricted to the seven British Society for Gynaecological Endoscopy (BSGE) endometriosis centres with the most complete follow-up

\begin{tabular}{|c|c|c|c|}
\hline & $\begin{array}{l}\text { Short term } \\
\text { (6 months)* }^{*}\end{array}$ & $\begin{array}{l}\text { Long term } \\
\left(2 \text { years) }{ }^{*}\right.\end{array}$ & Range \\
\hline \multicolumn{4}{|l|}{ Symptoms } \\
\hline Premenstrual pain & 10 & 7 & $(0-10)$ \\
\hline Menstrual pain & 10 & 9 & $(0-10)$ \\
\hline Non-cyclical pelvic pain & 10 & 5 & $(0,10)$ \\
\hline Deep dyspareunia & 10 & 5 & $(0,10)$ \\
\hline Cyclical dyschezia & 10 & 5 & $(0-10)$ \\
\hline Non-cyclical dyschezia & 7 & 4 & $(0-10)$ \\
\hline Low back pain & 10 & 5 & $(0-10)$ \\
\hline Bladder pain & 4 & 1 & $(0-10)$ \\
\hline Voiding difficulty & 1 & NS & $(0-10)$ \\
\hline $\begin{array}{l}\text { Frequent bowel } \\
\text { movements† }\end{array}$ & 2 & NS & $(0-4)$ \\
\hline $\begin{array}{l}\text { Urgent bowel } \\
\text { movements }\end{array}$ & 3 & NS & $(0-4)$ \\
\hline $\begin{array}{l}\text { Incomplete bowel } \\
\text { movements } †\end{array}$ & 2 & NS & $(0-4)$ \\
\hline Constipation† & 2 & NS & $(0-4)$ \\
\hline Blood in the stool $†$ & 2 & 1 & $(0-4)$ \\
\hline \multicolumn{4}{|l|}{ Quality of life $†$} \\
\hline $\begin{array}{l}\mathrm{EQ} \text { visual analogue } \\
\text { score }\end{array}$ & 0 & 55 & $(0-100)$ \\
\hline EQ5D usual activities & 1 & 1 & $(0-2)$ \\
\hline $\begin{array}{l}\text { EQ5D pain and } \\
\text { discomfort }\end{array}$ & 2 & 2 & $(0-2)$ \\
\hline $\begin{array}{l}\text { EQ5D anxiety and } \\
\text { depression }\end{array}$ & 2 & 1 & $(0-2)$ \\
\hline EQ5D mobility & 0 & 1 & $(0-2)$ \\
\hline EQ5D self-care & 1 & NS & $(0-2)$ \\
\hline EQ5D index & -0.594 & 0.65 & $(-0.594$ to 1$)$ \\
\hline
\end{tabular}

*The worst possible score that could be reported for all the missing postoperative data in order for the short-term (6 months) or longterm statistics in tables 3 and 4 to still be significant. (The test statistics that were not significant (NS) to start with have not been included.)

†A high EQVAS or EQ5D index score is associated with an improvement in QoL, whereas with all other QoL and symptom scores, a low value is associated with improvement.

EQVAS, EuroQol Visual Analogue Scale; EQ5D, EuroQol 5D; QoL, quality of life.

data and the method of collection have enabled a robust assessment of the risk of complications from this type of surgery. Precise estimates of efficacy of surgery and associated complication rates to inform clinical decision making have been lacking because relevant data available in the literature have been derived from small, singlecentre case series, ${ }^{910} 2021$ and in many such publications, the severity of endometriotic disease has been heterogeneous or not defined..$^{9} 102021$ We are aware of no other dataset that has examined the laparoscopic management of rectovaginal endometriosis when it is applied across a 
Table 6 Incidence of perioperative and postoperative complications

\begin{tabular}{|c|c|c|}
\hline & $\begin{array}{l}\text { Number of } \\
\text { patients }\end{array}$ & Incidence (\%) \\
\hline \multicolumn{3}{|l|}{ Peri operative complication } \\
\hline Haemorrhage $>1 \mathrm{~L}$ & 43 & 0.9 \\
\hline Ureteric injury & 24 & 0.5 \\
\hline Unexpected bowel injury & 28 & 0.6 \\
\hline Unexpected bladder injury & 17 & 0.4 \\
\hline Unexpected vascular injury & 10 & 0.2 \\
\hline Epigastric injury & 4 & 0.1 \\
\hline Conversion to laparotomy & 41 & 0.9 \\
\hline Colostomy & 9 & 0.2 \\
\hline Ileostomy & 14 & 0.3 \\
\hline $\begin{array}{l}\text { Unplanned removal of any } \\
\text { other organ }\end{array}$ & 11 & 0.2 \\
\hline Death & 0 & 0.0 \\
\hline $\begin{array}{l}\text { Total suffering any } \\
\text { perioperative complication }\end{array}$ & 220 & 4.7 \\
\hline \multicolumn{3}{|l|}{ Postoperative complication } \\
\hline Pelvic haematoma & 37 & 0.8 \\
\hline Pelvic abscess & 17 & 0.4 \\
\hline Urinary tract leak & 11 & 0.2 \\
\hline Bowel leak & 17 & 0.4 \\
\hline Urinary tract fistula & 2 & 0.0 \\
\hline Bowel fistula & 12 & 0.3 \\
\hline Severe sepsis & 10 & 0.2 \\
\hline Pulmonary embolism & 1 & 0.0 \\
\hline $\begin{array}{l}\text { Total suffering any } \\
\text { postoperative complication }\end{array}$ & 120 & 2.5 \\
\hline
\end{tabular}

Note that some patients suffered more than one complication.

country according to accepted best practice and within nationally approved guidance. ${ }^{6}$ Although there may be some units in the UK that do not submit data to the BSGE national database, this would be the exception for any National Health Service (NHS) unit carrying out large volumes of surgery as this has become the standard for commissioning of endometriosis services by NHS England.

The main limitation of our study relates to missing data from incomplete data entry, incomplete follow-up or uncompleted follow-up at closure of the study. We performed sensitivity analyses to explore the robustness of our results to incomplete follow-up. The results were stable, remaining significant in some cases even when symptomatic outcomes for those women with missing data were assumed to be the worst possible outcome. Furthermore, when we restricted analysis to the seven centres providing the most complete follow-up, the observed improvement in clinical symptoms and QoL were reproduced. Thus, it is unlikely that the impact of missing data would substantially alter clinical inferences on the efficacy of laparoscopic surgery for severe rectovaginal endometriosis.

We did not routinely collect data regarding body mass index that may impact on the incidence of surgical complications. Moreover, we did not collect data pertaining to reproductive history and fertility. Thus, we cannot comment on the impact of these factors on clinical outcomes. While the lack of these baseline data may limit the generalisability of our findings, we believe that our results are likely to remain externally valid in light of the magnitude and multicentre nature of our series. We evaluated symptoms relating to bowel function in addition to reporting pain symptoms known to be associated with endometriosis. However, condition-specific validated instruments assessing bowel symptoms in rectovaginal endometriosis are lacking. The data we provide are germane to the symptoms our patients with endometriosis complain of and as such we believe have face validity. We did not collect pain diaries where detail regarding the dose and pattern of analgesic use could be obtained. As we collected a large amount of data pertaining to pain, urinary and bowel function and the impact of symptoms of QoL, we adopted a pragmatic approach, limiting our enquiry to what type of analgesia was being used at the time of follow-up. In this way, we hoped to minimise the burden on the individual patient to respond enhancing completeness of data entry and follow-up. A final limitation relates to our use of historical control data from the same patients prior to surgical intervention, whereas the study would have benefited from a non-surgically

Table 7 Incidence of bowel complication related to the type of surgery performed on the bowel

\begin{tabular}{|c|c|c|c|c|c|}
\hline Type of bowel surgery & $\begin{array}{l}\text { Number of } \\
\text { operations }\end{array}$ & UBI (n (\%)) & Leak (n (\%)) & Fistula (n (\%)) & Total (n (\%)) \\
\hline No bowel surgery & 1740 & $6(0.3)$ & $3(0.2)$ & $2(0.1)$ & $11(0.6)$ \\
\hline Disc resection & 54 & $0(0.0)$ & $4(7.4)$ & $3(5.6)$ & $5(9.3)$ \\
\hline Segmental resection & 181 & $4(2.2)$ & $4(2.2)$ & $2(1.1)$ & $7(3.9)$ \\
\hline
\end{tabular}

Unexpected bowel injury (UBI) at the time of surgery. Leak=any bowel leak identified after primary surgery. Fistula formation is a late complication from surgery. 
treated control group. However, persuading a group of women with severe, refractory symptoms to continue with non-surgical treatment would be challenging.

Despite the complexity of the surgery, the overall reported perioperative and postoperative serious complication rates were relatively low at $4.7 \%$ and $2.5 \%$, respectively. Conversion to laparotomy occurred in less than $1 \%$ of cases demonstrating advances in acquisition of laparoscopic surgical skills, training and equipment. Excision of endometriosis in the posterior pelvis is a difficult surgical procedure, but the improved vision and precision that laparoscopy provides may improve completeness of excision and, in skilled hands, minimise the risk of complications. The ureteric injury rate of $0.5 \%$ was similar to those reported in smaller series from single centres and that associated with hysterectomy. ${ }^{22}$ Bowel trauma, namely unintended bowel injuries, leaking from bowel surgery and rectovaginal fistula formation, occurred in $1.2 \%$ of cases. In the context of the morbidity associated with rectovaginal endometriosis, its resistance to conventional medical treatments and the nature, complexity and efficacy of surgery, this rate of complications appears to be acceptable. Moreover, the rates of adverse events are comparable with other series from expert, single centres. ${ }^{20-22}$ The reported incidence of complications cannot, however, be used as indicative risk for patients who have care given in non-specialist endometriosis centres.

\section{Strengths and weaknesses in relation to other studies, discussing important differences in results}

The findings from the current study, which is the largest, multicentre series by far to be reported to date, are in keeping with those published from smaller, single-centre observational cohorts of women undergoing laparoscopic excision of deep endometriosis. Significant reductions in the intensity of chronic pelvic pain, dysmenorrhoea, dyspareunia and dyschezia were reported from series in the UK (57 and 137 women) ${ }^{1121}$ and Finland (22 women).$^{20}$ The majority of studies included in a recent extensive literature review of surgical treatment of deep endometriosis with colorectal involvement also reported improvements in pain and digestive symptoms as well as health-related QoL, although few studies reported this latter outcome. ${ }^{10}$ The size of the 49 included studies ranged from 4 to 283 cases, and the total number of operations evaluated was 1791 of which $679(38 \%)$ were rectal shaving procedures, 375 (21\%) disc resections and $737(41 \%)$ segmental resections. However, comparisons between studies were not possible because of inadequate reporting (many included studies reported 'overall improvement'), as well as inconsistent assessment, of clinical outcomes (eg, use of interviews, bespoke questionnaires and visual analogue scales). A systematic review of 34 articles describing 1889 segmental bowel resections also showed that the vast majority of women had significant improvement in chronic pelvic pain, dysmenorrhea, dyspareunia and dyschezia at 1 year. ${ }^{9}$
Safety, in addition to efficacy, is necessary to justify undertaking complex laparoscopic procedures for a benign pathology although a condition associated with substantial morbidity. Haemorrhage greater than $1 \mathrm{~L}$ was the most common perioperative complication occurring in $0.9 \%$ of cases. This rate is consistent with the available literature where haemorrhage requiring blood transfusion in complicated procedures $(0.3 \%-3.1 \%) \%)$ depending on the type of bowel surgery. ${ }^{10}$ Similarly, our perioperative and postoperative complication rates were comparable with the mean (range) of complications reported within a recent review of 49 case series ${ }^{10}$ : rectovaginal fistulae $0.3 \%$ versus $2.7 \%(0 \%-11 \%)$; enterostomy $0.5 \%$ versus $1.2 \%(0.0 \%-7.0 \%)$ and pelvic abscess $0.4 \%$ versus $0.3 \%(0.0 \%-4.0 \%)$. Complication data from a systematic review restricted to segmental bowel resection reported a $6.4 \%$ rate of severe bowel complications (leakage $1.9 \%$; fistula 1.8\%; severe obstruction $2.7 \%$ ), $2.5 \%$ rate of haemorrhage and $1 \%$ severe infection rate. ${ }^{9}$ Our ureteric injury rate of $0.5 \%$ is in keeping with the $1 \%$ reported in another series $^{21}$ as is the low observed prevalence of unintended bowel injury, major vascular injury and urinary tract fistula. ${ }^{20-22}$

\section{Meaning of the study: possible explanations and implications} for clinicians and policymakers

This study demonstrates that women having laparoscopic excisional surgery for rectovaginal endometriosis obtain significant symptomic relief, reduction in analgesia use and improvement in their QoL. These improvements are present at 6 months and sustained at 2 years, with the exception of voiding difficulty. The lack of improvement in this symptom may be explained by the fact that the presence of bladder endometriosis was not an inclusion criterion for this study and so voiding complaints may be an independent symptom. This study cannot predict outcomes more than 2 years postoperatively, but it is very encouraging that the worsening of symptom scores, analgesia use and QoL scores postoperatively (from 6 months to 2 years) is extremely small compared with the large clinical improvement seen initially.

Rectovaginal endometriosis sits between the vagina and the rectum and thus the most consistent symptom differentiating it from the pain associated with less severe and other forms of endometriosis or adenomyosis is pain on intercourse or 'dyspareunia'. In addition, in the more severe groups, pain on defecation is a common symptom due to the proximity to, or invasion of, the bowel. Thus, it is reassuring that the surgery resulted in a significant improvement in these symptoms and that the effect lasted for the 2 years of follow-up. One of the side effects of all surgery is scarring, and it is possible that a rectovaginal nodule of endometriosis excised may be replaced by scar tissue leading to a resumption of dyspareunia. These data would suggest that this was not the case. In addition, the long-term effects of surgery would suggest a lack of significant recurrence during the time scale of the study. 
Laparoscopic surgical excision of rectovaginal endometriosis appears to be effective in treating chronic pelvic pain, bowel and urinary symptoms and improving health-related QoL and has an acceptable major complication rate when performed in specialist surgical centres. Women with severe and refractory symptoms adversely impacting on their QoL should be offered laparoscopic surgical treatment in recognised endometriosis centres, where clinical outcomes are objectively audited. Commissioners of health services should restrict these highly complex surgical treatments to specialist centres to ensure an adequate case load and where expertise is demonstrated through provision of auditable clinical outcomes.

\section{Unanswered questions and future research}

Future studies should collect relevant baseline clinical data including comprehensive population characteristics, indications for surgery and fertility desires. Serial, standardised short-term and long-term follow-up will enhance comparability of data and a better evaluation of the longer term effects of laparoscopic interventions for deep rectovaginal disease on symptoms. Relevant and valid outcomes should be chosen with the help of patient involvement and should include valid collection of health-related QoL data. Such studies should be controlled where possible and ideally randomised to reduce selection bias. A randomised controlled trial comparing excisional surgery and diagnostic laparoscopy with the same number of ports is difficult ethically because women in the placebo arm would have been referred for specialist treatment and thus would be undergoing an unnecessary surgical intervention. Future studies should consider randomising between laparoscopic surgery and non-surgical therapy or alternatively compare different laparoscopic surgical interventions. The morbidity associated with rectovaginal endometriosis has a substantial economic impact due to the related reduction in activity both socially and in the work place. Thus, any future studies should include formal economic analysis to weigh the costs of surgical management against clinical, health service and societal gains.

\section{CONCLUSIONS}

This study presents by far the largest, multicentre rectovaginal endometriosis surgical series in the literature both in relation to clinical outcomes and complication rates. Deep endometriosis is associated with a high disease burden and limited access to effective medical and surgical treatment is a large unmet need. Indeed, half our study population had undergone previous surgery for endometriosis mostly in non-specialist centres. Women suffer with chronic pain and psychological symptoms that impair their QoL. Health services and the wider economy suffer through utilisation of substantial healthcare resources and restrictions placed on women's physical functioning that can lead to absenteeism and the inability to fulfil domestic and professional duties. ${ }^{23}$ The impact of endometriosis on QoL is well recognised ${ }^{2124-26}$ and well demonstrated in the current study where the median preoperative EQ global VAS score was only 55/100 and the median preoperative EQ5D index score only 0.525 . It is also widely acknowledged that surgical management of deep endometriosis involving the rectovaginal septum and/or bowel is technically challenging and can be associated with high rates of serious complications. However, women with deep endometriosis often have severe and refractory symptoms adversely impacting on their $\mathrm{QoL}^{6-8}$ justifying the need for effective surgical treatment even if associated with potentially serious complications.

Our large, multicentre dataset demonstrates significant improvements in a variety of pain and functional bowel symptoms from laparoscopic excisional surgery. The substantial and sustained improvements in pain symptoms and health-related QoL and reassuringly low rates of major perioperative and postoperative complications support this form of surgical treatment when conducted in specialist endometriosis centres. Future studies should include formal economic analyses to weigh the costs of surgical management against clinical, health service and societal gains.

Acknowledgements We are grateful to Jeremy Wright and Adam Moors, who designed and established the BSGE endometriosis centres database, and Dominic Byrne, who upgraded it to increase completeness of data collection and follow-up. We are grateful to the BSGE Scientific Advisory Group, who approve research on BSGE endometriosis centres database data and have scrutinised the manuscript. The members are: Professor Justin Clark (chair), Dominic Byrne, Oliver Chappatte, Alfred Cutner, Chris Guyer, Ertan Saridogan and Robert Richardson. We would like to thank and acknowledge all the BSGE endometriosis centres endocentre teams for contributing to this work by entering their data on the BSGE endometriosis database; they are listed alphabetically below with number of cases in brackets and name of lead gynaecologist.

Collaborators Aberdeen (99) Lucky Saraswat, ACEMIG (140) Shaheen Khazali, Altnergavin (36) Iris Menninger, Birmingham (58) Yousri Afifi, Castle Hill (103) Kevin Phillips, CEMIG (199) Saikat Banerjee, Chelsea and Westminster (258) Robert Richardson, Chester (88) Jeremy Hawe, Colchester (81) Barry Whitlow, Cornwall (257) Dominic Byrne, Croydon University hospital (57) Emmanuel Ofuasia Dewsbury/Elland (121) Ashwini Trehan, Dorset (57) Tyrone Carpenter, Endometriosis CaRe Oxford (164) Christian Becker, Exeter (23) James Clark, Exppect Edinburgh (52) Stuart Jack, Guy's and Thomas' (29) Kumar Kunde, Gynaecology.co (98) Alfred Cutner, Homerton (21) Chris Barnick, Imperial (75) Alan Farthing, James Cook Hospital (111) Graham Phillips, King's College (132) John Bidmead, Lancashire Women's and Newborn (31) Mohamed Abdel-Aty, Liverpool Women's (85) George Botros, Luton and Dunstable (35) Marlin Mubarak, Mid Yorks (46) Christian Kremer, Norfolk and Norwich University Hospital (53) Edward Morris, North Middlesex (34) Stanley Okolo, North West Pelvic Pain, Manchester (87) Edmond Edi-Osagi, Northwick Park Hospital (81) Kamel Shehata-Iskander, Penine (63) Gaity Ahmad, Plymouth (53) Jonathan Frappell, Preston Royal, Lancashire (31) Brice Rodriguez, , Queens University Hospital, Romford (27) Yemi Coker, Royal London (55) Elizabeth Ball, Royal Stoke (69) Gourab Misra, Southampton (160) Adam Moors, , Southmead (104) Sanjay Vyas, St George's Hospital (46) Thomas Ind, St Mary's Hospital, Portsmouth (143) Chris Guyer, St Michael's Hospital, Bristol (81) Caroline Overton, Stockport (60) Suku George, The Northern Endometriosis Team (49) Tony Chalhoub, Tunbridge Wells (146) Oliver Chappatte, University College London Hospital (302) Ertan Saridogan, University Hospital of Wales (80) Richard Penketh, UPMC Beacon (32) Moiad Alazzam, Whipps Cross Hospital (82) Funlayo Odejinmi, Winchester (21) Keith Louden, Wirral University Teaching Hospital (324) David Rowlands, Worcestershire (82) Jon Hughes.

Contributors DB maintained and upgraded the endometriosis database, designed the study and analysis, provided data and contributed to data interpretation and drafting of the manuscript. TC conducted the statistical analysis and data interpretation and contributed to drafting of the manuscript. PS designed the 
study, conducted literature and reference review and contributed to drafting of the manuscript. ES provided data and contributed to data interpretation and drafting of the manuscript. AC was responsible for set-up of the BSGE endometriosis Centres project, provided data and contributed to data interpretation and drafting of the manuscript. TJC chaired the BSGE endometriosis Centres Scientific Advisory Group, designed the study and contributed to data interpretation and drafting of the manuscript and is the guarantor on behalf of the BSGE endometriosis centres research group.

Funding The BSGE is a registered charity and paid for the development and maintenance of the endometriosis database. The BSGE provided limited funding for one author to perform background research and literature review (PS) and one author (TC) to undertake statistical analysis. There was no direct research funding apart from the BSGE financial support to set up and administer the database and employ a statistician and a research fellow. Each hospital that contributed data acted as a study sponsor in that they agreed to collection of data from their respective endometriosis centre and it being stored on the BSGE endometriosis centres database for research.

Competing interests All authors with the exception of TC are members of the BSGE.

\section{Patient consent Obtained.}

Ethics approval All patients gave written consent to have their data stored on the BSGE database and for its subsequent use in scientific research and publication. Written consent was obtained each time symptoms and QoL data were collected. The database is managed in accordance with the data protection act, data are encrypted and the database is hosted by a third party that is paid by the BSGE for this service. The BSGE designed and developed the database. The BSGE is a charity that raises funds from its membership and scientific meeting sponsorship. All hospitals where clinicians enter data are informed in writing by the BSGE to the chief executive officer and medical director about the patient data storage on the BSGE endometriosis database to ensure their agreement. Two authors were funded to carry out initial research (PS) and to perform statistical analysis on the data (TC).

Provenance and peer review Not commissioned; externally peer reviewed.

Data sharing statement TJC on behalf of all other named authors affirms that the manuscript is an honest, accurate and transparent account of the study being reported; that no important aspects of the study have been omitted; and that any discrepancies from the study as planned have been explained. The full dataset is available from the corresponding author at t.j.clark@doctors.org.uk

Author note We confirm that all authors had full access to all of the data (including statistical reports and tables) in the study and can take responsibility for the integrity of the data and the accuracy of the data analysis.

Open Access This is an Open Access article distributed in accordance with the Creative Commons Attribution Non Commercial (CC BY-NC 4.0) license, which permits others to distribute, remix, adapt, build upon this work non-commercially, and license their derivative works on different terms, provided the original work is properly cited and the use is non-commercial. See: http://creativecommons.org/ licenses/by-nc/4.0/

(C) Article author(s) (or their employer(s) unless otherwise stated in the text of the article) 2018. All rights reserved. No commercial use is permitted unless otherwise expressly granted.

\section{REFERENCES}

1. Dunselman GA, Vermeulen N, Becker C, et al. ESHRE guideline: management of women with endometriosis. Hum Reprod 2014;29:400-12.

2. Koninckx PR, Martin DC. Deep endometriosis: a consequence of infiltration or retraction or possibly adenomyosis externa? Fertil Steril 1992;58:924-8.
3. Vercellini P, Crosignani PG, Somigliana E, et al. Medical treatment for rectovaginal endometriosis: what is the evidence? Hum Reprod 2009;24:2504-14.

4. Fedele L, Bianchi S, Zanconato G, et al. Gonadotropin-releasing hormone agonist treatment for endometriosis of the rectovaginal septum. Am J Obstet Gynecol 2000;183:1462-7.

5. Busacca M, Somigliana E, Bianchi S, et al. Post-operative GnRH analogue treatment after conservative surgery for symptomatic endometriosis stage III-IV: a randomized controlled trial. Hum Reprod 2001;16:2399-402

6. Jerby BL, Kessler H, Falcone T, et al. Laparoscopic management of colorectal endometriosis. Surg Endosc 1999;13:1125-8.

7. Redwine DB. Laparoscopic en bloc resection for treatment of the obliterated cul-de-sac in endometriosis. J Reprod Med 1992;37:695-8

8. Emmanuel KR, Davis C. Outcomes and treatment options in rectovaginal endometriosis. Curr Opin Obstet Gynecol 2005;17:399-402.

9. De Cicco C, Corona R, Schonman R, et al. Bowel resection for deep endometriosis: a systematic review. BJOG 2011;118:285-91.

10. Meuleman C, Tomassetti C, D'Hoore A, et al. Surgical treatment of deeply infiltrating endometriosis with colorectal involvement. Hum Reprod Update 2011;17:311-26.

11. Kent A, Shakir F, Rockall T, et al. Laparoscopic Surgery for Severe Rectovaginal Endometriosis Compromising the Bowel: A Prospective Cohort Study. J Minim Invasive Gynecol 2016;23:526-34.

12. Saridogan E, Byrne D. The British society for gynaecological endoscopy endometriosis centres project. Gynecol Obstet Invest 2013;76:10-13.

13. Adamson GD. The modern role of reproductive surgery. Clin Obstet Gynecol 2011;54:710-9.

14. Adamson GD, Pasta DJ. Endometriosis fertility index: the new, validated endometriosis staging system. Fertil Steril 2010;94:1609-15.

15. Tuttlies F, Keckstein J, Ulrich U, et al. [ENZIAN-score, a classification of deep infiltrating endometriosis]. Zentralb/ Gynakol 2005;127:275-81.

16. Anon. Revised American Society for Reproductive Medicine classification of endometriosis: 1996. Fertil Steril 1997;67:817-21.

17. EuroQol Group. EuroQol-a new facility for the measurement of health-related quality of life. Health Policy Amst Neth 1990;16:199-208.

18. Rabin R, de Charro F. EQ-5D: a measure of health status from the EuroQol Group. Ann Med 2001;33:337-43.

19. Dolan P, Gudex C, Kind P, et al. A Social tariff for Euroqol: results from a UK general population survey discussion paper 138. York: Univerity of York, 1995.

20. Setälä M, Härkki P, Matomäki J, et al. Sexual functioning, quality of life and pelvic pain 12 months after endometriosis surgery including vaginal resection. Acta Obstet Gynecol Scand 2012;91:692-8.

21. Garry R, Clayton R, Hawe J. The effect of endometriosis and its radical laparoscopic excision on quality of life indicators. BJOG 2000;107:44-54.

22. Mabrouk M, Montanari G, Guerrini M, et al. Does laparoscopic management of deep infiltrating endometriosis improve quality of life? A prospective study. Health Qual Life Outcomes 2011;9:98.

23. Soliman AM, Yang H, Du EX, et al. The direct and indirect costs associated with endometriosis: a systematic literature review. Hum Reprod 2016;31:712-22.

24. Dubernard G, Piketty M, Rouzier R, et al. Quality of life after laparoscopic colorectal resection for endometriosis. Hum Reprod 2006;21:1243-7.

25. Redwine DB, Wright JT. Laparoscopic treatment of complete obliteration of the cul-de-sac associated with endometriosis: longterm follow-up of en bloc resection. Fertil Steril 2001;76:358-65.

26. Dubernard G, Rouzier R, David-Montefiore E, et al. Use of the SF-36 questionnaire to predict quality-of-life improvement after laparoscopic colorectal resection for endometriosis. Hum Reprod 2008;23:846-51. 